Data sources Cochrane Hepato-Biliary Group controlled trials register, Cochrane Controlled Trials Register, MEDLINE, LILACS, SCI Expanded, Embase and http://clinicaltrials.gov/ until January 2010; reference lists of relevant articles and authors were searched. Review methods The methodological approach outlined in the Cochrane Handbook was followed. Randomised trials comparing antibiotics with placebo, no intervention, non-absorbable disaccharides, another antibiotic or any other active treatment for HE were included. The primary outcome measures were no improvement of HE and all-cause mortality.

Results Twenty-eight studies were included in this review; most had a small sample size and were methodologically of a low quality. Two studies $(\mathrm{N}=132)$ compared antibiotics with placebo or no intervention, reporting no differences in primary outcomes between groups. Twelve studies $(\mathrm{N}=708)$ compared antibiotics with nonabsorbable disaccharides, reporting no difference in all-cause mortality ( $R R=1.11$ (95\% CI 0.60 to 2.07$)$ ) but a marginally significant reduction in the risk of "no improvement" (RR 0.83 (0.69 to 1.00$)$ ). In sensitivity analysis, this finding was significant in trials evaluating chronic encephalopathy ( $R R 0.50$ (0.28 to 0.89)) and in trials with co-administration of cathartic agents (RR 0.82 (0.67 to $0.99)$ ). Ten trials $(\mathrm{N}=336)$, compared different antibiotic regimens, most evaluating rifaximin and neomycin. Rifaximin reduced the risk of no improvement compared to neomycin, but not significantly so (RR 0.61 (0.34 to 1.09$)$ ).

Conclusions Insufficient evidence exists to support or refute the use of antibiotics for HE. Antibiotics were slightly superior to nonabsorbable disaccharides at improving $\mathrm{HE}$, with borderline significance and possible effect modification.

\section{P2-77 BEHAVIOUR PROBLEMS AND PREVALENCE OF ASTHMA SYMPTOMS AMONG BRAZILIAN CHILDREN}

doi:10.1136/jech.2011.142976i.12

\begin{abstract}
${ }^{1,2} \mathrm{C}$ Feitosa, ${ }^{*} \mathrm{D}$ Santos, ${ }^{3} \mathrm{M}$ B do Carmo, ${ }^{1} \mathrm{~L}$ Santos, ${ }^{4} \mathrm{C}$ Teles, ${ }^{2} \mathrm{~L}$ Rodrigues, ${ }^{1}$ M Barreto. 'Institute of Collective Health, Federal University of Bahia, Salvador, Bahia, Brazil; ' London School of Hygiene and Tropical Medicine, LSHTM, London, UK; ${ }^{3}$ Institute of Psychology, University of São Paulo, São Paulo, Brazil; ${ }^{4}$ Institute of Statistics, State University of Feira de Santana, Feira de Santana, Bahia, Brazil
\end{abstract}

Introduction Asthma is the most common chronic disease in childhood and has been designated a public health problem due to the increase in its prevalence in recent decades, the amount of health service expenditure it absorbs and an absence of consensus about its aetiology. The relationships among psychosocial factors and the occurrence, symptomatology, and severity of asthma have recently been considered. There is still controversy about the association between asthma and child's mental health, since the pathways through which this relationship is established are complex and not well researched. This study aims to investigate whether behaviour problems are associated with the prevalence of asthma symptoms in a large urban centre in Latin America.

Methods Cross-section study of 869 children between 6 and 12 years old, residents of Salvador, Brazil. The International Study of Allergy and Asthma in Childhood (ISAAC) questionnaire was used to evaluate prevalence of asthma symptoms. The Child Behaviour Checklist (CBCL) was employed to evaluate behavioural problems. Results $19.26 \% \quad(n=212)$ of the children presented symptoms of asthma. We found good evidence of an association between behaviour problems and asthma symptoms (PR: 1.53 ; 95\% CI 1.13 to 2.08 . $\mathrm{p}=0.007$ ) and this association remained statistically significant after adjustment for sex, age, maternal education, income, parental asthma, minor psychiatric disorders in the mother, Alcohol use, allergens in dust and smoking (PR: 1.43 ; 95\% CI 1.10 to 1.85. $\mathrm{p}=0.01$ ). Conclusion These results suggest an association between behavioural problems and paediatric asthma, and support the inclusion of mental healthcare in the provision of services for asthma morbidity.

\section{P2-78 QUALITY OF LIFE IN BRAZILIAN WOMEN WITH BREAST CANCER: ASSOCIATION WITH THE SOCIAL ENVIRONMENT}

doi:10.1136/jech.2011.142976i.13

${ }^{1}$ D B Ferreira, ${ }^{* 1} \mathrm{R}$ Koifman, ${ }^{2} \mathrm{~A}$ Bergmann. ${ }^{1}$ National School of Public Health, Oswaldo Cruz Foundation, Rio de Janeiro, Brazil; ${ }^{2}$ National Cancer Institute, Rio de Janeiro, Brazil

Introduction The incidence of breast cancer is increasing in Brazil. Breast cancer is a major cause of morbidity and mortality with major social and epidemiological impact. The aim of this study is to evaluate the association between social support before treatment for breast cancer and quality of life (QOL) 6 months after starting treatment.

Methods Eligible participants were women who were treated for incident breast cancer in the National Cancer Institute between June 2009 and March 2010. EORTC OLQ-C30 was used to measure QOL. MOS-SSS was used to assess social support prior to treatment. Bivariate analyses of dichotomous variables were carried out and ORs with 95\% CI were presented.

Results 195 women were evaluated. Good emotional support was a protective factor for overall health (OR 0.42 (95\% CI 0.23 to 0.79 )) and scale functionality (OR 0.36 (0.18 to 0.71$)$ ). Information support was associated with better global health (OR 0.41 ( 0.22 to $0.76)$ ), functionality (OR 0.35 (0.18 to 0.70$)$ ) and symptoms scale (OR 0.38 (0.18 to 0.77$)$ ). Women who reported good emotional support were $70 \%$ less likely to report general health problems 6 months after starting treatment $(0.13-0.65)$.

Conclusion The results of this study suggest that a good social environment prior to treatment is positively associated with $\mathrm{QOL}$ 6 months after starting treatment in women with breast cancer, underscoring the importance of assessing social environment.

\section{P2-79 THE IMPACT OF SOCIAL ENVIRONMENT ON QUALITY OF LIFE IN ELDERLY WOMEN WITH BREAST CANCER IN BRAZIL}

doi:10.1136/jech.2011.142976i.14

${ }^{1} \mathrm{D}$ B Ferreira, ${ }^{*} \mathrm{R}$ Koifman, ${ }^{2} \mathrm{~A}$ Bergmann. ${ }^{1}$ National School of Public Health, Oswaldo Cruz Foundation, Rio de Janeiro, Brazil; ${ }^{2}$ National Cancer Institute, Brazil, Rio de Janeiro, Brazil

Introduction Studies suggest that social support is associated with improved health outcomes in cancer patients. The relationship between social environmental and health outcomes in elderly Brazilian women with breast cancer has not been investigated. This study aimed to explore the association between social support prior to treatment in a reference centre in Brazil and quality of life (QOL) in elderly women and women under 60 years of age with breast cancer.

Methods This study included women with breast cancer who started oncology treatment at National Cancer Institute between June 2009 and March 2010. The Medical Outcomes Study-Social Support Survey was used to assess social support prior to treatment. EORTC QLQ-C30 and BR23 module were used to measure QOL. Bivariate analyses of dichotomous variables and independent variables were carried out and RRs with CIs of $95 \%$ presented.

Results 195 women were examined of whom 46.2\% (90) were elderly. Older women who reported good emotional support had lower risk of presenting the worst score of global health (RR 0.69 (95\% CI 0.55 to 0.88$)$ ), functionality (RR 0.59 (0.38 to 0.91$)$ ) and 